\title{
DEVELOPMENT OF SMART PAINTING MACHINE USING IMAGE PROCESSING
}

Atif Saeed

Faculty, Department of Mechatronics Engineering, SZABIST, (Pakistan). E-mail: m.atif@szabist.edu.pk ORCID: https://orcid.org/0000-0003-4369-2388

Hussain Muslim Mithaiwala

Student, Department of Mechatronics Engineering, SZABIST, (Pakistan). E-mail: hussain.muslim53@gmail.com ORCID: https://orcid.org/0000-0001-6284-6237

Ammar Iqbal Hussain

Student, Department of Mechatronics Engineering, SZABIST, (Pakistan). E-mail: ammararsi48@gmail.com ORCID: https://orcid.org/0000-0003-2031-8393

Tulsi Kumar

Student, Department of Mechatronics Engineering, SZABIST, (Pakistan). E-mail: tulsikumar199600@gmail.com ORCID: https://orcid.org/0000-0002-8848-0533

Recepción: 04/09/2020 Aceptación: 02/10/2020 Publicación: 14/12/2020

\section{Citación sugerida:}

Saeed, A., Mithaiwala, H.M., Hussain, A.I., y Kumar, T. (2020). Development of smart painting machine using image processing. 3C Tecnología. Glosas de innovación aplicadas a la pyme, 9(4), 95-1 19. https://doi.org/10.17993/3ctecno/2020. v9n4e36.95-119 


\section{ABSTRACT}

Painting is a process in automotive industry that takes about $10 \%$ of the total time consumed per vehicle. Currently, the painting lines in several automotive industries like Toyota motors or General motors is largely being automated after implementation of robotic arms. However, according to the study done, this automation is restricted to paint main body or chassis only, the coating and painting on small vehicle parts is still done manually. This paper represents the study conducted on local Toyota motor plant located in Karachi where the need of automating the small parts painting line evolved to increase production. The designed system is based on 3axis gantry mechanism which locates the position of parts to be painted on moving conveyor through computer vision, reach the coordinates, paints autonomously and forwards it to buffer station for drying. The computer vision is introduced to minimize the human interference and make the line semi-autonomous. The system was tested for correct image processing and at the end algorithms was correctly identifying and locating $90 \%$ of the parts. Moreover, the system was tested for one week in a laboratory environment and it was observed that production rate was increased to $2 \%$ as compare to their previously available data.

\section{KEYWORDS}

Computer Vision, Autonomous Painting, CNC, Automobile, Mechatronic System, Python. 


\section{INTRODUCTION}

Today, the modern industrial revolution is on its way towards advancements. The industrial revolution is taking over the entire world briefly. One of the major goals of today's industrial revolution is to adopt automation. Almost in every sector such as military, agriculture, automobile, manufacturing and design and development etc. Presently, automation is widely being implemented and used in the industrial sector all over the world, this is done in order to increase the efficiency and productivity of the manufactured goods. The automobile sector is also using automation to perform various task in the cars body manufacturing, assembly and paint shops in order to increase their per day productivity and efficiency of the cars. But however, the industrial automation is not implemented and used in Pakistan at its maximum pace. Thus, a lot of task are done manually, this affects the efficiency and productivity of the produced parts. In Pakistan, especially in the automobile sector, painting procedures are not fully carried out by robots, instead paid labors (humans) are also employed in painting small parts such as mirror covers, mud flaps, door handles, spoiler and fog lamps etc. And also, automatic painting is adopted only by factories and industries which produce large lot sizes of same type/variant of parts (Saeed et al., 2019).

Thus this paper discusses about the possible feasible solution which will help shift the small scale industries and the automobile industries to implement modern industrial automation by placing robots in their production line in order to increase their productivity and efficiency of produced goods.

Currently, Pakistan is not able to completely not able to adopt the industrial revolution along with the modern automation as because the initial capital cost is way too much for which small industries can't invest in it. The other major reason is that most of the robots can paint only one part variant at a time, thus industries not producing large lot sizes of same part variant are unable to get automatic painting being carried out in their industry. Another problem caused by this is the human health issues, as painting process contains release of harmful chemical fumes which ultimately affects human health. Also human painted parts are sometimes not up to the mark as the paint coating are uneven, thus the parts are not 
passed from quality check departments which results in reducing the productivity as well as efficiency of the parts produced by the industry. Thus, all these reasons affect the productivity and efficiency of the industrial produced goods (Amin \& Saeed, 2018).

Our idea is to introduce a Smart Painting Machine which will be capable of painting various geometry parts at a time. By this our aim is to introduce the concept of automatic painting in small scale industries who cannot afford costly automatic painting machines within their vicinity. We basically derived the idea of automatic painting from the automobile industries where still small parts such as mud flaps, door handles, spoilers, fog lamps etc. are painted by labors.

The key feature of this machine is basically its programming part which waves off the requirement to burn/run different program for different geometrical part. The programming embedded with the smart painting machine is basically based on image processing. A camera is mounted in the workspace of the painting machine which takes the real-time image of the part and then the image is processed. After processing the image, its geometry is detected by applying edge detection and contour detection. This results in identifying the object geometry by which the object coordinated are detected. Thus the part is painted as per its geometry. This features allows small lots of various geometrical parts to be painted with a single machine and single program. Thus, it's very beneficial for industries as it saves a lot of capital investment.

As far as human health is concerned, this machine follows all the safety protocols. The painting workspace is enclosed by a glass/acrylic frame house to eliminate the chances of spilling out paint particles as well as escape of any painting chemical fumes. Thus a lot health issues like coughing, sneezing, nausea and breathing issues are eradicated. 


\section{LITERATURE REVIEW}

Authors, in this paper, plans to introduce a process for advance mechanize spray painting of unknown parts. The machine made by this experiment will be very useful for painting of unknown shape. The time consumed by this machine is very less as compared to manually, (hand) painting. It will, save expenditure such as labor cost and the total cost of painting any jobs (Swarakar et al., 2018).

Thakar and Vora (2014) in his paper gives essential knowledge about mini and large scaled industries manufacturing parts are painted for protecting from rust, so the spray method consumes large amount of time and paint which required the workers which are skilled emerged with the application. Robotic painting techniques is not applicable for large efficiency so the rise in such method have to be made which is affordable, have accuracy and precision, consumes minimum time for the coating of the part so objective has to developed in such a manner that the mechanism which coat the part with the dipping and baking process having semi-automated techniques which is up to the required mark and which can be valuable for mini and large scale factories (Thakar \& Vora, 2014).

Author in this research highlights some key features through the system test, it is fact that the design of intelligent robot have many advantage not only has good painting effect but also has high efficiency which can only at least 2-6 minutes to draw the simple cartoon images and also take no more than 15 minutes to draw complex portrait images. We also provided an illustrative example to show our required results (Feng et al., 2017).

Abdellatif (2012) in his research describes the design, construction and working of an Automatic wall painting robotic machine. This visionary and remarkable design of a robot which is movable to be used for painting interior walls of residential building or offices has been described. Robot has a roller that is fed with liquid paint and keeps contact with the wall surfaces. The robot has advance option that helps the roller to scan vertically as well as horizontally to the painted walls. The robot has advance technology that can adjust itself in front of the wall (Abdellatif, 2012). 
Naticchia et al. (2007) in his research, shows that mechanical painting can be not only be done to upgrade production and allows quality checking. The robotic arm application with high precision and accuracy is required. An automatic system to convert to the normal coordinates of the liquid colors to be reproduce the moveable speed of the robot's end tool and valve opening and closing end of the mixing board. The maximum work shall be probably required to get high resolution (Naticchia et al., 2007) hence also the productivity of the construction industry must be improved, while preserving its labour from hazardous job sites. Such requirements can be accomplished by the adoption of robotized products, which, however, need to be quickly developed and marketed. In this paper, first the issue of a new miniature laboratory for developing lightweight and well-coordinated robotized systems is pursued, then a novel robot device for high quality multi-colour interior wall painting carried by a robot arm is developed and successfully tested. Thanks to the new 1:6 scaled down laboratory and its six degree of freedom robot arm on an hexapod for horizontal moves, we tested the opportunity to introduce also in the building sector miniature robots that can change the ergonomics standardly adopted by construction workers. It is analyzed how and why switching from full size to miniature robots is convenient in construction. In addition, a new system adding further features to robotized painting has been conceived. Our new multi-colour spraying end-tool was developed and fixed on the robot arm, in order to be able to reproduce coloured artworks. Finally, a methodology to reproduce colours from digital format of artzoorks is presented, showing how accurate and efficient is this new robotized spraying device. miniature painting robot, scaled down laboratory, multi-colour spraying end tool. Ata and Eleyan (2017) in their research present his work on articulated robots like these robots are widely re-known by basically automobile company commercials and robot dance application. SCARA (Selective-Compliance-Articulated-Robot-Arm) robots are also re-known for their usage and proliferate in industries from 1970's. Two kind of robot articulated and SCARA robot's combination to gather linear and rotary motion accomplishing in formation for complex tasks (Ata \& Eleyan, 2017). 
In this research paper goal was to learn the system for coating and painting tasks carried out in automobile repair and then change manual painting by robot painting. So the most important study, was for skilled spray gun handling for automobile repair painting which were observed and compared with those with little or no experience. The spray gun movements of the experts were characterized by longer length, longer time, higher speed, and narrower swing range, compared with the non-experts. The results were collected and accordingly the spray gun movement was set (Ikemoto et al., 2015).

Authors in this paper presents a research on Image Processing that can be directed to Machine Learning and the process of computing can identify pattern of high diverse parts. Machine learning is very close and like computing statistics that consist of spam filter optical character identification searching engines and computer vision. Their extensive arrays of knowledge observed (algorithms) to reduce destruction noise such linear filter of Gaussian-based algorithm. Algorithms can remove certain kind of grain noise destruction from a picture. Because every pixel of picture in setting to mean values in its environment the normal variations tested by the grain are reduced (Wiley \& Lucas, 2018).

Authors in this project through his studies, successfully identified the part from the background picture used for color process is required to remove the background by 1st filer grayscale filtering is the second step and finally by Circular Hough Transform (CHT) and binary testing for part that is in circular object detection. Using of color processing is used as it is powerful process to identify the part as it is in normal color process it has a lot of information as human eyes can do. For the grayscale filter it filters the (pixel and smoothness) the picture to the edge clear. In last CHT is required to detect the parts which are circular and total number is displayed (Hussin et al., 2012).

Authors in this research paper formulates that it is not possible to consider a single process for all type of images, nor can all process perform well for types of image. The background subtraction process identify parts with noise destruction and output is not accurate and precise. The object behind object is not recognized. During identification of part when any other thing come before the part problem occurs. The image cannot be recognized if the position of camera is not accurate and object in picture 
is not snapped properly (Jain \& Chadokar, 2015)Object recognition is a work of searching a selected object in an image or video sequence. Object detection plays a key role in image processing, It helps in searching of any particular object Object recognition is use to detect a particular object from a series of other objects, sometimes shadow and background images becomes problem in detection. Skull detection technique is use resolving such kind of problems. Here we deals with differ sort of object detection techniques and modes of multiple object detection for a image.

The authors, in this paper, through his knowledge and work proposes an algorithm that has been proved to meet the requirement of object detection without using the color feature in an automatic robot. The proposed algorithm specially relies on two main process that emphasize on shape identification and feature extraction that follows.

The first method is edge detection and line-oriented method to performing contour extraction, which results in object detection in very less or no time. Next, the second method is a geometric moment that captures and computes the global features of the objects. Both the process is well assured in image processing, however, a mixture of both is a novel method in this study and has been proven to precisely detect static and moving object under illumination variety (Dewi et al., 2019)such as the lengthy process to calibrate color, color fading, and others. Nonetheless, the need of such application that does not necessarily rely on color information has seen a hike due to the mentioned issues. In fact, some of the desirable solutions are those that take less computation time, as well as those that provide higher accuracy and scalability for a large number of objects in a scene. One application that requires such solution is in a game playing by autonomous robot. This paper suggests a novel patch carried by autonomous robots with relevant detection algorithm using contour detection and geometric moment without using the color feature. 


\section{METHODOLOGY}

\subsection{DESIGNING PHASE}

\subsubsection{HARDWARE DESIGNING}

So initially, it is not recommended to go towards hardware fabrication directly. It is more desirable to first virtually design the hardware on a CADCAM Software, such as SolidWorks. Mostly, the thinking and fabricating part does not go hand to hand simultaneously as it's necessary that what we think can be implemented and fabricated practically, this leads to loss of time and capital. So, in order to check our design ideas and its practical fabrication feasibility, we do the designing work first.

So, in the designing phase, we first started with our conveyor. First the conveyor frame structure of dimensions 70in x 20in x 3in was made. To understand it, it is basically like a table structure. Now, in order to run make the conveyor bed, we attached 2 rollers at each end of the conveyor frame. By attaching two rollers, it was observed that the conveyor belt be stable while moving as when the part comes in the middle of the conveyor belt while travelling, the belt will sag downwards due to no roller in the middle and this will cause irregular movement of the conveyor belt. Thus, we added a third roller in the middle of the conveyor frame.

We, now, added a white rubber belt of $2 \mathrm{~mm}$ thickness over the rollers to form a conveyor bed. Three double slotted pulleys of were mounted on each on the three rollers in order to interconnect the rollers with each other so that movement of the rollers are in sync. These pulleys are interconnected 2 rubber V-Belt and the third belt of size relates to a pulley and with a D.G Geared Motor of 30Nm torque (24V$3 \mathrm{~A}$ ) in order to drive the conveyor. The part to be painted will be placed on the conveyor which will help in transporting the part to and from the painting work area. The conveyor design is given below in Figure 1. 


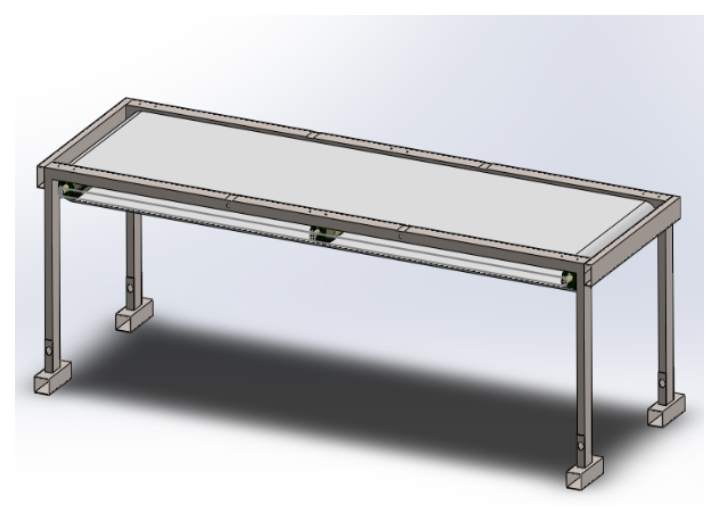

Figure 1. Conveyor Design.

After the completion of the Conveyor Design or the Part Transporting Mechanism, we started to design the CNC Mechanism which is the Painting Mechanism of our Machine. The overall size of the CNG mechanism is 24in x 20in x 24in. Each of the 3 axes of the CNG machine is made of square shaped 2.5" 1 " mild steel pipe. The main axis mechanism is formed of a ball screw of length $500 \mathrm{~mm}$ and $8 \mathrm{~mm}$ size of double threads. Each of the two ends of the ball screw is connected in the 8mm bearing which is mounted inside bearing housing.

Now, we will screw an $8 \mathrm{~mm}$ double threaded nut over which an aluminum cube is fixed which acts as a traveler. All these things are fixed to form a single axis structure. We replicate this structure four time to form $\mathrm{X}, \mathrm{Y}$ and $\mathrm{Z}$ axis individually ( $\mathrm{X}$ axis is made from 2 of these structures). We mount individual Stepper Motor on each axis in order to rotate the ball screw so that the aluminum cube mounted over a nut move linearly back and forth. Each axis motion is limited/restricted by attaching a limit switch at its both ends. This helps in restricting the axis motion in forward or backward direction, so all the 3-axis does not collide with each other. The painting spray gun is mounted on the Z-axis of the CNC Mechanism. The Design of CNC Mechanism is given below in Figure 2. 
Figure 2. CNC Mechanism Design.

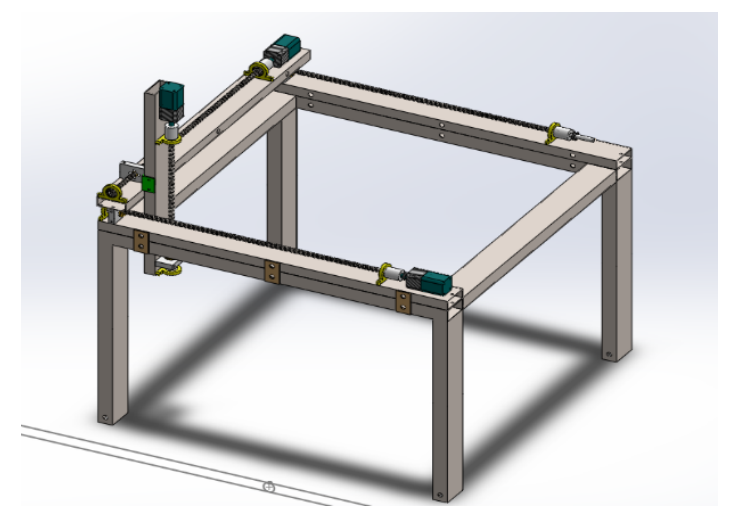

This CNC Mechanism is mounted on the conveyor in the middle, thus the area under the CNG mechanism is the painting working area. Ball screws are used to move the 3-axis of the GNC mechanism. These ball screws are rotated with the help stepper motor and stepper drives are also used. The painting spray gun is mounted on the Z-axis of the GNG Mechanism.

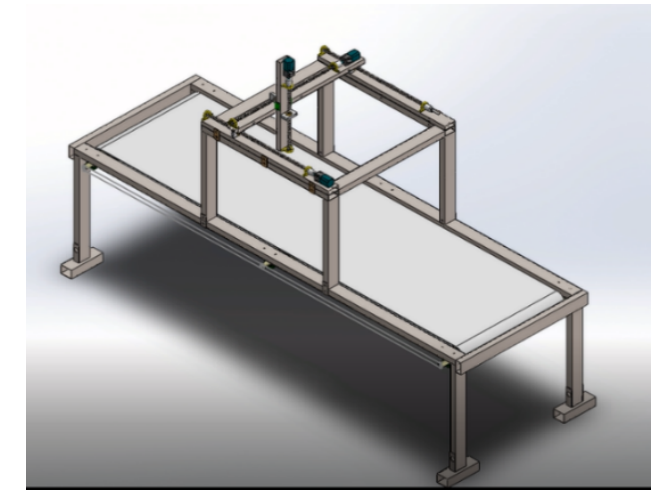

Figure 3. Smart Painting Machine Complete Hardware Design.

\subsubsection{ELECTRONIC CIRCUITRY DESIGNING}

Apart from the hardware of the machine, the Brain which will help in driving the functionality of this machine is the Electronic Circuitry. Basically, the electronic Circuit of this machine needs to be designed 
exclusively for itself. The readily available circuits can't be integrated with one another to drive this painting machine, instead its circuit needs to be designed. The main task required by the circuitry is to:

1. Drive and Control the motion of the Conveyor.

2. Drive and Control the motion of the Painting Mechanism (i.e. 3-axis GNC Mechanism).

3. Assist the Image Processing Program in execution so that the part Geometry can be identified, and it's coordinated can be extracted in order to move the painting mechanism to perform the painting task.

Thus, in order to accomplish this task we chose Raspberry Pi Microcontroller which will control the entire circuitry. Raspberry $\mathrm{Pi}$ will be powered by a $5 \mathrm{~V}$ supply input which is fed from a $24 \mathrm{~V}$ Power Supply after connected a buck converter in series in order to step-down the voltage. Also, the conveyor motor will be powered by this power supply and its programmable controlling will be handled by the switching of a relay which is connected to the raspberry pi microcontroller. The Raspberry Pi Camera Module will be connected to the microcontroller which through its assistance will capture the image of the part which will enter the workspace to be painted. Also the Image Processing Software, which is developed on OpenCV to detect the part, identify it and calculate its geometrical coordinates will also be executed by this microcontroller. Also, after the extraction of the parts center coordinates, these coordinates will be via Encoder and TTL IC will be fed to Arduino.

The Arduino Microcontroller will take in the centroid coordinates of the part (X, Y, Z) and then will drive the painting mechanism motors in order to paint the part. The motion of the axis is limited by Limit Switches.

The Designed Circuit for Smart Painting Machine is given in Figure 4 and Figure 5 below. 


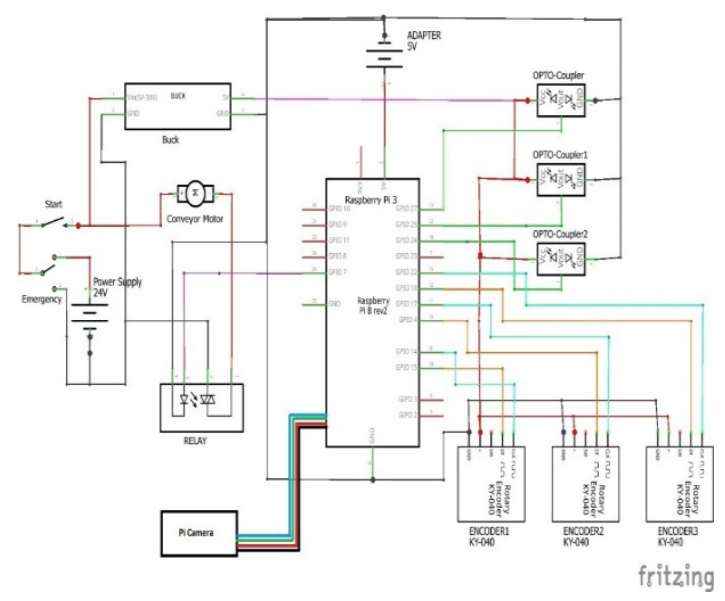

Figure 4. Design of Circuit 1.

Figure 5. Design of Circuit 2.

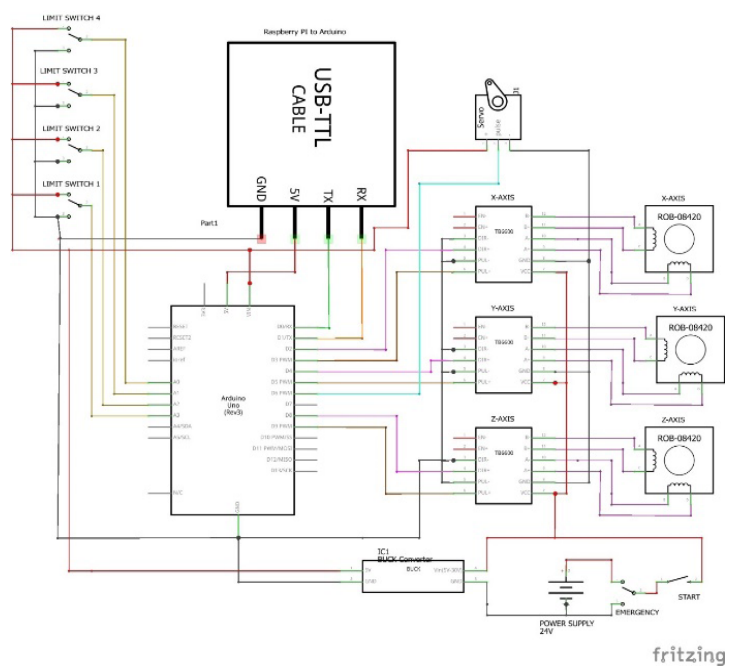

\subsubsection{SOFTWARE DESIGNING}

The heart of this Smart Painting Machine is its Software. The software is basically termed as heart because the sole purpose which makes this machine smart is the image processing program. We thought of applying image processing in this machine as to get rid of uploading new programing from every 
new part and forbid the idea of keeping/placing the part to be painted in a fixed place or to be fixed in its respective jigs. Image processing will help in determining the part orientation on the conveyor bed and will calculate the part coordinates and will command the motor to move the axis respectively in order to paint it. To implement this idea, we had to first check that whether his idea is working or is practically feasible to implement on a machine to calculate the part coordinates on real-time basis. Thus, for developing, testing and final implementation of this program, we chose to do this task using Python.

The camera which will be taking the part image will be mounted over the workspace. Once the part enters the workspace, IR sensor will indicate the microcontroller to stop the conveyor and then the part image will be taken and processed and then the part will get painted and then the conveyor will move, and the part will depart the workstation. So, to implement the software idea, we made program which works as follows:

First the part image is read by the program. After reading the image, filters of Erosion (process which removes pixel from boundary of the object in an image) and Dilation (process which adds pixel to boundary of the object in an image) are implemented. Then the object in the image is subtracted from its background and then thresholding is applied on the image. After this Canny Edge Algorithm (which detects edges of the object with noises suppressed at the same time) is applied and then the Counter detection is applied (which joins the curve of all the continuous points along with boundaries of same pixel intensity).

This enable the program to calculate the center of the object and its dimension. After this the coordinated of the image is found. Then the motor steps are calculated, and this is passed on to the XYZ Algorithm which then controls the motor movement of the CNG axis. The below Figure 6 shows the Software design flowchart. 


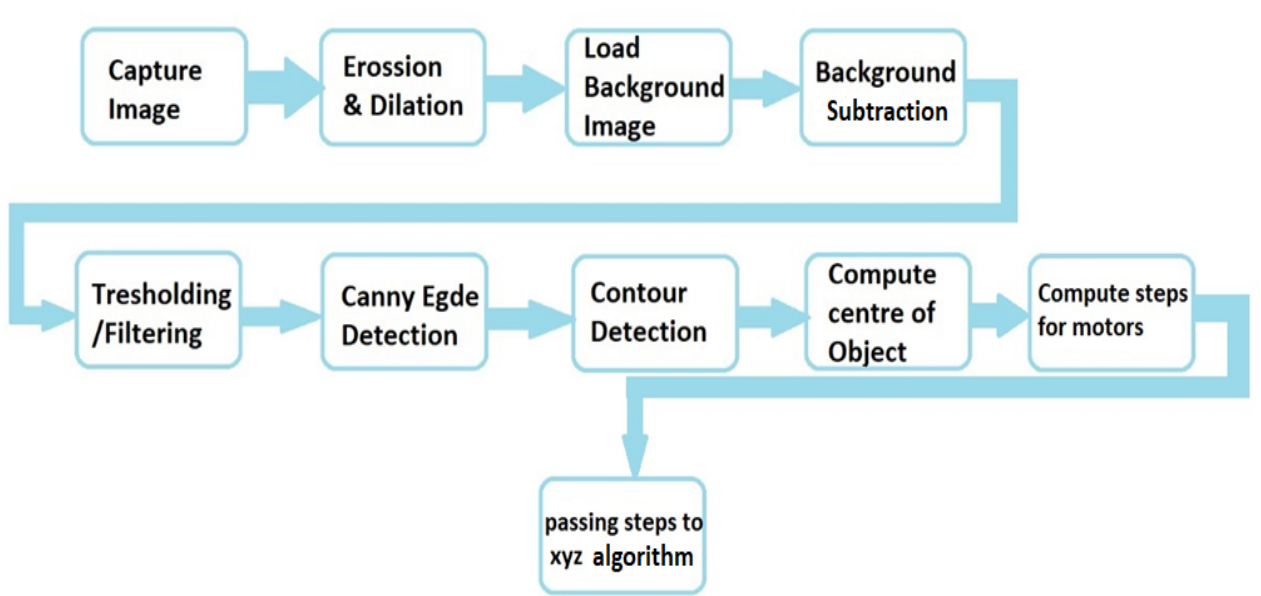

Figure 6. Software Flowchart.

\subsection{IMPLEMENTATION VIA VIRTUAL SIMULATION OF THE PAINTING MACHINE}

\subsubsection{SOLIDWORKS DESIGN SIMULATION AND ANALYSIS OF THE HARDWARE}

As the Hardware was not able to get complete due to the pandemic situation, what we did that we designed and made a complete CAD Model of our hardware (which was to be fabricated) with exact configurations so that the hardware can be tested with respect to its structural properties and working principle.

SolidWorks Software was used to make the CAD Model of the Hardware and its Tools were used to obtain graphical results of the Structural Rigidity Tests which includes Stress and Strain graphs of the critical hardware element. Also, Motion Analysis was performed of the working hardware and respective graphical results were calculated and portrayed in order to confirm the smooth working of the hardware and its motor while on full loading conditions. 
This will not only confirm the proper hardware working but will also help in analyzing the motor selection done in our project based on the interrupted working of the motors under loading conditions. Also the velocity of the GNC mechanism, i.e. the speed with which its axis moves can also be obtained.

\subsubsection{IMAGE PROCESSING PROGRAMMING SIMULATION USING JUPYTER}

As briefed before, the Image Processing Program is used to detect the part geometry (i.e. the part which will be placed on a conveyor and enter the workspace) so that part can be painted as per its geometrical figure. This image processing programming was done on Python.

This task was to be performed on real-time basis that as the part would enter the workspace, the camera mounted on the workspace would capture the image of the part placed on the conveyor and then image processing program would process this image and compare it with original part image in the directory and would classify the part and detect its geometry and center coordinates so that the part could be painted as per its geometry. However, due to unavailability of the hardware of this project, the testing of this image processing programming was performed on Jupyter.

The testing results were perfect (as predicted) and the part was classified, its geometry was identified, and its center coordinates were perfectly calculated. This data found by Image Processing Program is further useful to paint the part as per its geometry.

\subsubsection{SIMULATION OF ELECTRONIC CIRCUITS AND PAINTING MECHANISM USING ARDUINO AND PROTEUS}

At first, Raspberry Pi was to be used as a Controller in order to run the entire Painting Machine and its Electronics. As we moved towards Simulation, due to unavailability of the raspberry pi simulator, we used Proteus and Arduino in order to simulate the painting mechanism and other electronics of our project. The Part Coordinates obtained from the Image Processing is now fed into the Arduino Programming (used to drive the Painting Mechanism as per the part geometry in order to perform Painting). This Arduino coding was interfaced to Proteus on which we had made the entire Electronic 


\section{Circuitry of the Painting Machine.}

As we ran the Arduino coding, so the part coordinates interpreted by the coding was used in the simulation of the circuitry in Proteus. The Smart Painting Machine Circuitry in the Proteus Simulation worked sequentially. As the part is placed on the conveyor, Sensor 1 detects the part presence and the Conveyor Motor starts.

Now as the part enters workspace, Sensor 2 detects the part and stops the Conveyor Motor and after getting the part coordinates via image processing the Painting Mechanism start moving as per the geometry and the part is painted. After the painting process is completed, the conveyor starts again and the part starts to move along the conveyor until it reaches the end of the conveyor where Sensor 3 detects the parts and stops the conveyor motor so that the painted part can be picked up by the human operator/labor.

\subsection{SMART PAINTING MACHINE WORKING PRINCIPLE}

Initially, conveyor will be stationary i.e. it won't be moving. There is an IR sensor mounted on the input side of the conveyor frame. The task of this IR sensor is to signal the conveyor's motor driver circuit that the part is placed at the input side of the conveyor. As the part is placed the conveyor, motor starts, and the belt begins to rotate over the conveyor rollers and the part moves forward towards the workspace i.e. Painting Mechanism.

Now as the part moves inside the workspace, I.R sensor mounted inside the workspace signals the conveyor motor driver circuit to stop its motion and simultaneously it also informs the Main Controller of the Painting Machine (i.e. Raspberry Pi) that the part is now present inside the workspace. As now the part is stationary inside the workspace, the camera mounted in the workspace captures the image of the part and sends it to the main controller where further the part geometry is calculated by the program. As soon as the part geometry is calculated the main controller dispatches X Y Z Algorithm to the motor 
driving circuit of CNC mechanism.

The GNC mechanism has a Painting Spray gun mounted on its z-axis. CNC mechanism or the painting mechanism moves as per the X Y Z Algorithm and the paint gun paints the part as per its geometry by moving about the coordinates of the part edges. Here the Limit Switches which is connected to each and every axis of the CNC machine restricts the extra axis motion and helps in avoiding axis collision, thus there is no chance of distortion occurrence during the painting procedure. Also, the Encoder Sensors mounted on the motors shaft keeps the stepper motor control drive updated about motors direction of rotation, position and speed. After the completion of part painting or we can say that after the complete execution of the X Y Z Algorithm, the painting mechanism moves at its home position and the camera captures a photo of the part and sends it to the main controller to check the painting work. After this machine controller sends a signal to the motor driver circuit of the conveyor motor to start its motion which in turn takes the path outside of the workspace and the part keeps on moving and until it reaches the end of the conveyor bed where IR sensor detects the part arrival and inform the motor driver circuit of the conveyor to stop its motion so that the part can be picked up by the user. As the part is picked up, the sensor signals the motor driver circuit of the conveyor that it can start its motion and thus the cycle of painting the next part continues. Also, for Safety purpose, Human/Obstacle Detection Sensors are mounted outside the workspace which signal the main controller to stop all the operations at once if any human detection is made near the workspace. This promotes human safety. 


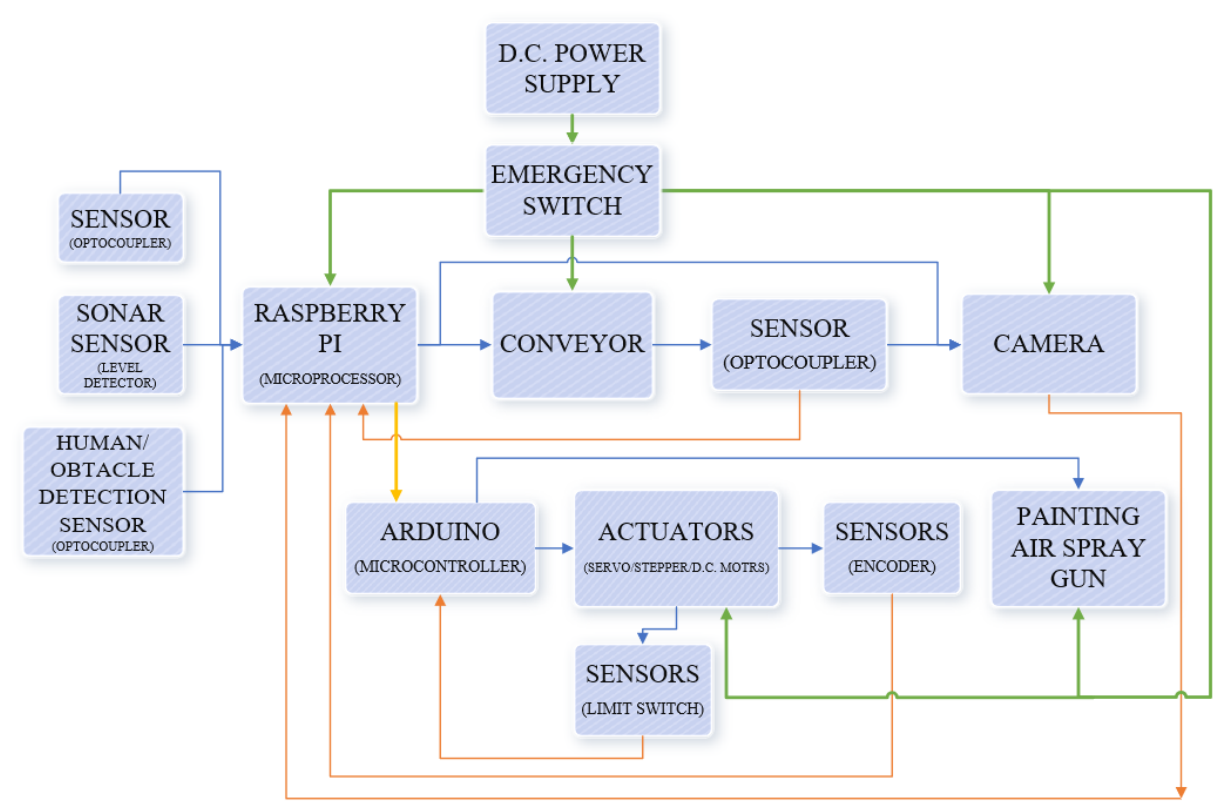

Figure 7. Standard Operating Procedure of Smart Painting Machine.

\section{RESULTS}

\subsection{HARDWARE SIMULATION RESULTS}

Smart Painting Machine's Hardware was designed and simulated on SolidWorks. The hardware was fabricated asper the designed model. During the designing phase the material used for fabrications of the machine, ball-screw material and design configurations, driving motors, conveyor belt material and other things were all kept the same which we were going to use for the real hardware fabrications. This was done so to check the proper working of hardware during the simulation phase and rectify and error/ problem (if any) so that it can be tackled during the designing phase only and no problems are caused during fabrications. To further ensure the hardware structural rigidity reliability, the critical element of the hardware (the component which bears maximum load during operation) was checked for its maximum stress it can handle and its factor of safety (FOS) was checked. It was found out that the Yield 
Stress of ball screw is $350 \mathrm{MPa}$ and due to the load exertion, the Stress caused on ball screw is not more than 66.04MPa. Also, the Factor of Safety is 2.7 which means that the ball screw is very far away from fracture.

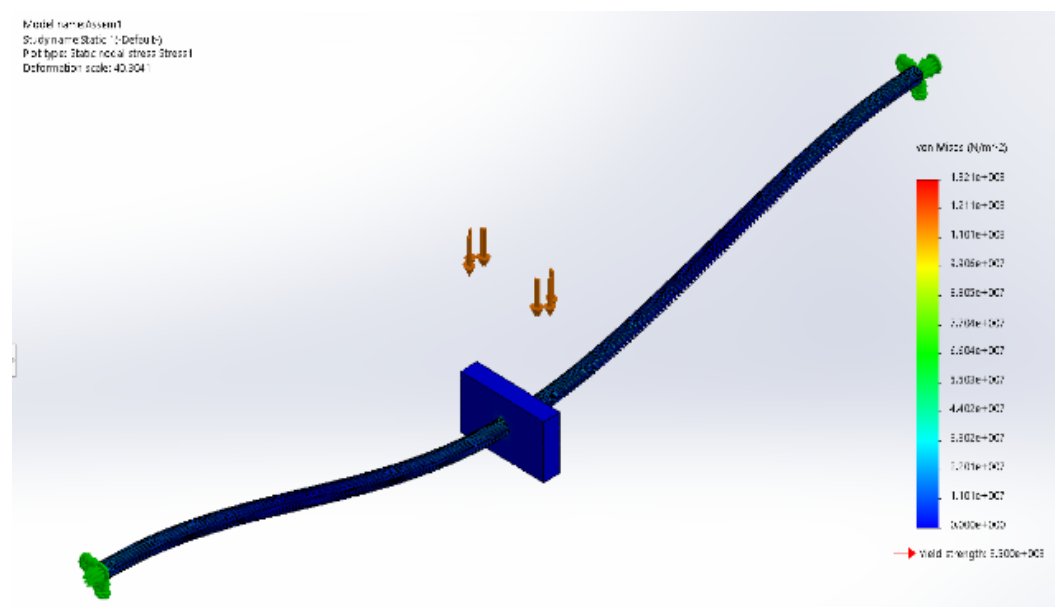

Figure 8. Ball Screw Stress Analysis.

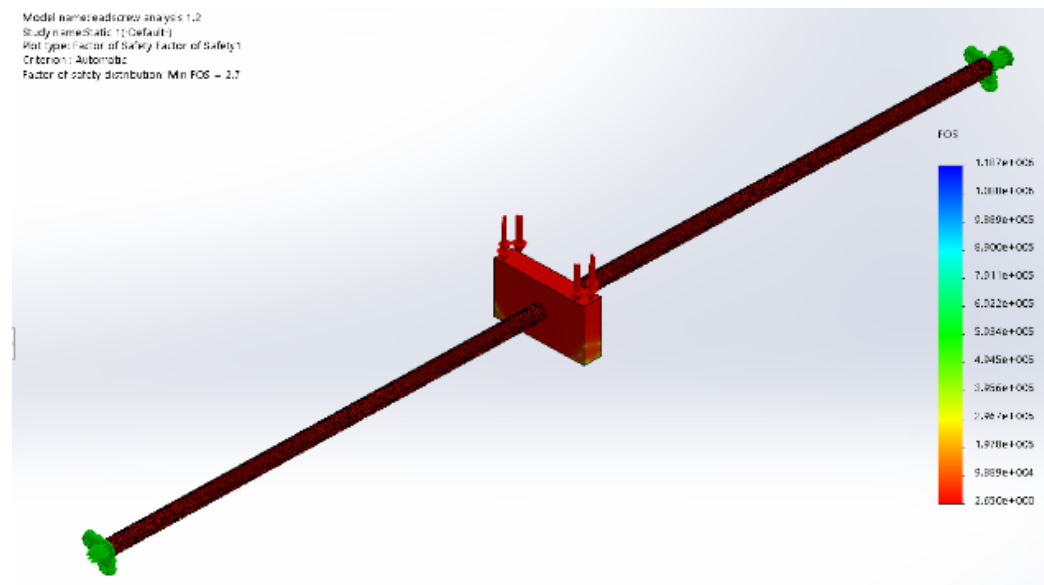

Figure 9. Ball Screw Factor of Safety. 


\subsection{IMAGE PROCESSING PROGRAM SIMULATION}

After successfully making the Image Processing Program of Object Detection and Classification and Simulating the Program on Jupyter, the desired results were obtained. The Resulting Images of the Parts shows perfect geometry detection of the part which is to be painted and its center coordinates are given. Almost 11 different parts were tested, result of some parts are given below.

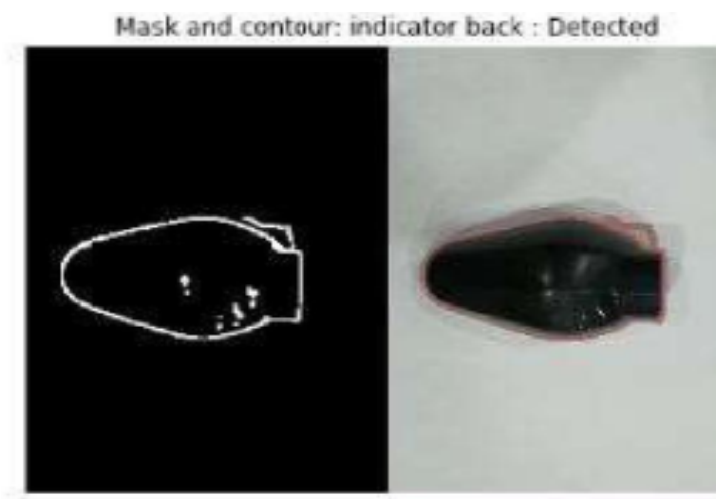

Figure 10a. Indicator Back cover- Object Detection.

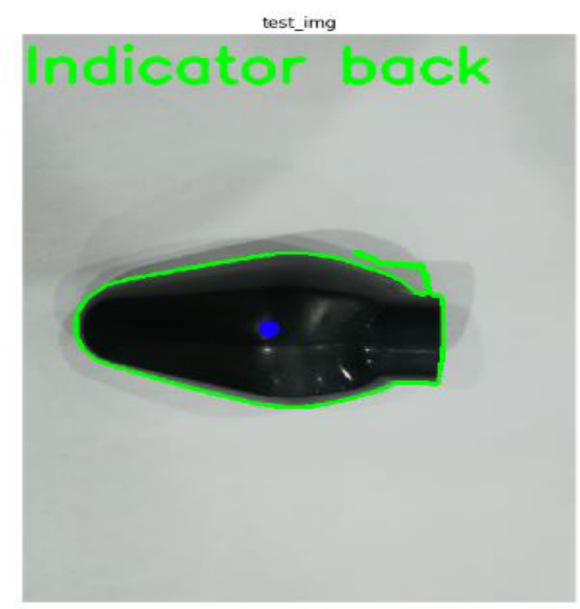

Figure 10b. Indicator Back cover- Object Classification. 


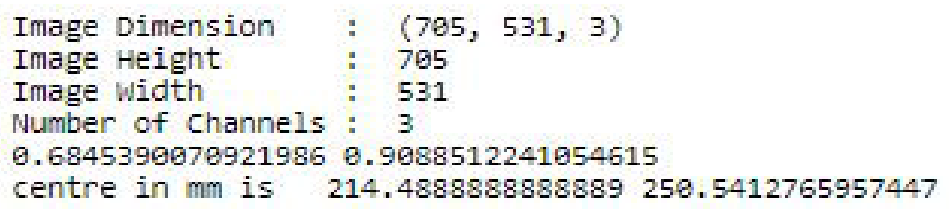

Figure 10c. Indicator Back cover- Object Center Coordinates.

\section{CONCLUSION}

By analyzing and observing the simulated results we can conclude that the Smart Painting Machine is working as per the requirement and is performing the desired job efficiently. The Simulated Results of the hardware the shows feasibility of functionality and robustness of the machine itself. The designing part helps us determine whether the components that are to be incorporated in the machine structure will work as per the requirement and how the maximum loading conditions will affect the working of the painting mechanism. The results obtained from the testing of the Hardware CAD Model further proves that the Hardware Design is up-to the mark in all aspects of robustness and functionality.

Also, by observing the simulation results of the Image Processing Program, we can conclude that the objects which will be placed on the conveyor will be correctly identified by the Developed Program using Image Processing. We have developed this program using Python OpenCV. The results obtained are very much accurate to that of the objects in the sample/saved images of the parts in the directory. We have also identified that whatever the orientation of the part on the conveyor, still the program will able to identify the part correctly. Also, we during testing of this program also observed that the size of the real-time captured image of the part (zoomed-in or zoomed-out photograph) but still the program will detect and classify the part. However, there is a limitation that the angle of capturing the image of the sample and test image should be same.

The simulation of the Electronic Circuitry of the Smart Painting Machine was executed very well. This is because the Designed Circuitry of the Painting Machine fulfills all the requirements of which assists in smooth operation of the painting machine. 


\section{REFERENCES}

Abdellatif, M. (2012). Design of An Autonomous Wall Painting Robot Design of An Autonomous Wall Painting Robot. In First Interna1onal Symposium on Socially and Technically Symbio1c Systems. https:// www.researchgate.net/publication/236659040_Design_of_An_Autonomous_Wall_Painting_ Robot

Amin, I., \& Saeed, A. (2018). 5.10 Wireless Technologies in Energy Management. Comprehensive Energy Systems, 5, 389-422. https://doi.org/10.1016/B978-0-12-809597-3.00524-1

Ata, A. A., \& Eleyan, A. (2017). An Autonomous Robotic Cell for Painting Applications. Acta Press. https:// doi.org/10.2316/P.2017.848-014

Dewi, D. A., Sundararajan, E., Prabuwono, A. S., \& Cheng, L. M. (2019). Object detection without color feature: Case study Autonomous Robot. International Journal of Mechanical Engineering and Robotics Research, 8(4), 646-650. https://doi.org/10.18178/ijmerr.8.4.646-650

Feng, X., Chen, L., Zhou, W., \& Huang, Z. (2017). Research on the Design of Smart Paint Robot. DEStech Transactions on Materials Science and Engineering, c(smne), 1-5. https://doi.org/10.12783/ dtmse/smne2016/10590

Hussin, R., Juhari, M. R., Kang, N. W., Ismail, R. G., \& Kamarudin, A. (2012). Digital image processing techniques for object detection from complex background image. Procedia Engineering, 41, 340-344. https://doi.org/10.1016/j.proeng.2012.07.182

Ikemoto, S., Liao, M., Takai, Y., Goto, A., Nishimoto, H., \& Hamada, H. (2015). Impact of Operator Experience on Spray Gun Handling for Automobile Repair. Procedia Manufacturing, 3, 488-494. https://doi.org/10.1016/j.promfg.2015.07.212 
Jain, S., \& Chadokar, S. (2015). A Object Detection in Image Processing: A Review. International fournal of Electrical, Electronics and Computer Engineering, 4(2), 26-29. https:/ / www.researchtrend.net/ijeece/ ijet21/ijetnew/4\%20SHRISHTEE\%20JAIN.pdf

Naticchia, B., Giretti, A., \& Carbonari, A. (2007). Set up of an automated multi-colour system for interior wall painting. International Journal of Advanced Robotic Systems, 4(4), 407-416. https://doi. $\mathrm{org} / 10.5772 / 5666$

Saeed, A., Haroon, K. M., Talha, M., \& Thebo, T. M. (2019). Frictional Analysis of different coatings on MS material in order to increase the life of machine element. In 3rd International Symposium on Multidisciplinary Studies and Innovative Technologies (ISMSIT), 1-6. https://doi.org/10.1109/ ISMSIT.2019.8932769

Swarakar, J. R., Belkhade, R. D., Dalal, V. G., Ghode, G. D., Maskar, S. L., Fulmali, S. M., \& Shelki, S. B. (2018). Design and Fabrication of Automatic Spray Painting Machine. International Fournal of Advance Research and Innovative Ideas in Education, 4(2), 1388-1394. http://www.ijariie. com/AdminUploadPdf/DESIGN_AND_FABRICATION_OF_AUTOMATIC_SPRAY_ PAINTING_MACHINE_ijariie7676.pdf

Thakar, D., \& Vora, C. P. (2014). A Review on Design \& Development of Semi-Automated Colour Painting Machine. Journal of Engineering Research and Applications, 4(4), 58-61. https://www. academia.edu/7548109/A_Review_on_Design_and_Development_of_Semi_Automated_ Colour_Painting_Machine

Wiley, V., \& Lucas, T. (2018). Computer Vision and Image Processing: A Paper Review. International Fournal of Artificial Intelligence Research, 2(1), 22. https://doi.org/10.29099/ijair.v2i1.42 
